\title{
Why collect individual-level vaccination data?
}

\author{
Writing team for the Public Health Agency of Canada/Canadian Institutes of Health Research \\ Influenza Research Network Vaccine Coverage Theme Group
}

Previously published at www.cmaj.ca

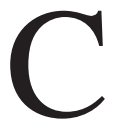

anada has been developing a national network of immunization registries since 1998. ${ }^{1}$ In March 2004, in response to the lessons learned from the outbreak of SARS (severe acute respiratory syndrome), the federal government called for the creation of a pan-Canadian system to collect, analyze and disseminate public health surveillance data for the management of infectious diseases. ${ }^{2}$ The resulting system, Panorama, will include an immunization registry that likely will prove valuable for responding to public health emergencies and evaluating vaccines. However, not all provinces and territories are adopting Panorama, and the staggered rollout of the system in the participating provinces will not occur until between 2010 and 2012. ${ }^{2}$ It has not been possible for all jurisdictions to mobilize quickly enough to collect data on pandemic H1N1 influenza vaccination. However, it is apparent that alternative — and for some jurisdictions, temporary — strategies to collect individual-level data are needed to ensure optimal responses against all vaccine-preventable diseases, including future pandemics.

\section{Importance of individual-level data}

Public health authorities routinely collect vaccination data to facilitate program planning and delivery (e.g., to ensure appropriate vaccine distribution), to evaluate program performance (e.g., to assess whether population-level vaccine coverage is achieved) and to conduct research (e.g., to conduct vaccine- and disease-related mathematic modelling). Although such data can be collected through surveys, they are vulnerable to bias and generally not available in a timely manner. In contrast, registries capture comprehensive data that can quickly provide accurate and precise vaccination information at both the individual and population level. In addition, although aggregate data may suffice for logistic outcome measures (e.g., number of vaccine doses administered), the collection of individual-level data facilitates appropriate scheduling of vaccine doses, tracing and notification in the event of safety concerns, accurate measurement of coverage and an evaluation based on health outcomes.

Individual-level data provide the detail required for scientifically rigorous studies of vaccine effectiveness (e.g., cohort and case-control studies) $)^{3}$ and safety (e.g., self-matched case series design). ${ }^{4}$ Researchers are forced to use the less ideal ecologic study designs when only aggregate data are available. ${ }^{5}$ The linking of accurate and reliable data on the vaccina-

\section{Key points}

- Individual-level vaccination data are critical for the timely evaluation of vaccine coverage, effectiveness and safety at the population level.

- Electronic collection of data allows real-time analyses and rapid response.

- Information systems are not used routinely across Canada for electronic collection of individual-level vaccination data.

- Nationally coordinated efforts to capture individual-level vaccination data, although not feasible for the 2009 pandemic H1N1 influenza vaccinations, can help in the fight against all vaccine-preventable diseases.

tion status of individuals to data on outcomes and adverse events from laboratory, disease surveillance and health administrative databases can help us understand our response to the current pandemic and prepare for future pandemics.

If safety issues are identified for specific vaccine lots, notifying recipients is possible only if the lot number and patient contact information are available. This will be especially important with new vaccines, for which fewer safety data are available. ${ }^{6}$ Given the potentially large number of vaccinated people, it would be extremely time-consuming and less accurate to search charts, consent forms or other paper-based vaccination records.

With individual-level vaccination data, reminders can be sent to recipients when more than one dose is required. This is particularly important because the effectiveness of the vaccine is reduced when doses are not spaced at appropriate intervals.

Individual-level data can also be used to evaluate the effectiveness of the vaccination program. Public health authorities can use rates of coverage to help modify delivery strategies. Aggregate data allow estimates of vaccine coverage for large areas (e.g., province, region); individual-level data, which provide estimates on a more granular geographic scale, will result in rapid and more precise monitoring of coverage. This increased resolution can help with operational planning and local intervention.

Although assessments of vaccine coverage in specific age groups can be performed with either aggregate or individual-

Members of the Public Health Agency of Canada/Canadian Institutes of Health Research Influenza Research Network Vaccine Coverage Theme Group are listed at the end of this article.

CMAJ 2010. DOI:10.1503/cmaj.091515 
level vaccination data, the linking of individual-level data with data from health administrative databases may permit an estimate of coverage, albeit not in real time, in priority highrisk groups.

The benefits of a robust system to collect individual-level data can extend to all vaccines - an important factor given the diminished public confidence in vaccination in recent years. ${ }^{?}$

\section{Collection methods}

Individual-level vaccination data can be collected manually (e.g., with pen and paper), electronically or both. Paperbased recording of vaccination information in a patient's chart or via a consent form is simple and inexpensive. However, it limits timely use of the data; information such as vaccine coverage based on age or high-risk group must be extracted manually through a resource-intensive and errorprone process. Electronic methods, such as the proposed Panorama system, permit real-time analyses and decisionmaking with the best evidence.

The most common hybrid approach involves recording data on paper at the point of care and manually entering the record in an electronic repository. This approach generally delays the availability of the data. An alternative is to use customized forms that are scanned electronically once completed, which preserves the simplicity of capturing data on paper without losing the benefit of timely electronic availability. Other hybrid approaches include accessing alternative sources of information, such as claims for physician billing.

Even as a hybrid solution, the considerable benefits of an electronic system include the ability to retrieve individuallevel data for operational and program-related planning of vaccine delivery and the potential to link the data to other databases to efficiently evaluate vaccine safety and effectiveness. These functions are important for pan-Canadian surveillance when vaccination programs are implemented.

\section{Barriers to collecting electronic data}

Although there are clear benefits to collecting individual-level vaccination data electronically, there are also potential barriers.

Considerable government financing is required to establish and maintain robust immunization registries. The federal government has already invested \$135 million in Panorama, and more resources will be required for its implementation, support and maintenance. ${ }^{2}$ Some jurisdictions that have already invested in Panorama may not want to commit additional resources solely for collecting vaccination data until the system is implemented. However, there are less costly and less technologically intensive ways to collect individual-level vaccination data that could be integrated into Panorama in the future. Options include hybrid systems such as paper scans or lightweight electronic systems for mass immunization clinics.

Barriers related to the accessibility and use of electronic individual-level data will be challenging to overcome. In some jurisdictions, vaccinations are conducted by public health staff, institutional occupational health staff and physicians. Combining data from various providers into a centralized registry requires considerable coordination. Systems such as Panorama address this issue by adopting pan-Canadian and internationally established standards for integrating electronic health records. For example, the use of HL7 messaging ensures semantic interoperability between applications and jurisdictional infrastructures. ${ }^{2}$ However, the creation of a national immunization registry is particularly difficult because of differing provincial privacy legislation, the absence of overall governance structures and the need for interprovincial access agreements. ${ }^{1} \mathrm{Nev}-$ ertheless, a network of provincial and territorial immunization registries is desirable. Centralized registries present their own issues, requiring additional financial and human resources to manage the large quantity of individual-level data being stored.

Ensuring the privacy and security of personal health information in electronic systems is another potential barrier. Safeguards such as encryption of personal identifiers, password protection and auditing of data access can readily address this concern.

Inaccuracies in collected vaccination data affect their optimal use for operational planning and research. However, modern systems can validate data at the time of entry, when errors can be corrected quickly. Accuracy can also be increased through data links that permit real-time verification with electronic health records. Such links also increase the usefulness of the data for research.

Finally, human barriers could affect the willingness of jurisdictions to adopt an electronic system to collect individual-level vaccination data, particularly in an emergency setting, such as a pandemic. Front-line vaccination staff may not be comfortable with computer technology and could resist using information systems, perhaps believing that they would increase their workload. However, studies testing the use of electronic data entry have shown that, after an initial - and often brief - training period, the time spent entering data decreases and overall efficiency improves. ${ }^{8}$ Methods for rapid data entry, such as swipe-card technology for patient demographic data, and prepopulated registries can save time.

\section{Conclusion}

The collection of individual-level vaccination data at the point of delivery is important for several reasons, including the ability to rapidly assess vaccine coverage, effectiveness and safety. A coordinated pan-Canadian effort for collecting and sharing individual-level data has not been feasible for the 2009 pandemic H1N1 influenza vaccinations. Still, the efforts of provinces and territories to amass such data within their jurisdictions will be tremendously valuable in the fight against all vaccine-preventable diseases.

\section{This article has been peer reviewed.}

\section{Competing interests: None declared.}

Disclaimer: The views expressed in this article do not necessarily reflect those of the Public Health Agency of Canada or the Canadian Institutes of Health Research.

Acknowledgements: The authors thank the Canadian Association for Immunization Research and Evaluation for providing networking assistance. They also thank Canada Health Infoway (an independent, federally 
funded, not-for-profit organization tasked with accelerating the development of electronic health records across Canada) for providing information about Panorama.

Funding: The activities of the Public Health Agency of Canada/Canadian Institutes of Health Research Influenza Research Network Vaccine Coverage Theme Group are supported by an operating grant from the Public Health Agency of Canada and the Canadian Institutes of Health Research.

\section{REFERENCES}

1. Canadian consensus conference on a national immunization records system. Can Commun Dis Rep 1998:24:137-40.

2. Final report: 2009 performance evaluation of the Canada Health Infoway Public Health Surveillance Program. Ottawa (ON): KPMG LLP; 2009. Available: www2.infoway-inforoute.ca/documents/Infoway-PHS\%20Evaluation-Final-March $\% 202009 \% 20-\% 20$ EN.pdf (accessed 2009 Nov. 25).

3. Mangtani P, Cumberland P, Hodgson CR, et al. A cohort study of the effectiveness of influenza vaccine in older people, performed using the United Kingdom general practice research database. J Infect Dis 2004:190:1-10.

4. Farrington $\mathrm{CP}$, Nash J, Miller E. Case series analysis of adverse reactions to vaccines: a comparative evaluation. Am J Epidemiol 1996;143:1165-73.

5. Kwong JC, Stukel TA, Lim J, et al. The effect of universal influenza immunization on mortality and health care use. PLoS Med 2008;5:e211.

6. Supply and safety issues surrounding an H1N1 vaccine. Lancet 2009;374:358.

7. Cooper LZ, Larson HJ, Katz SL. Protecting public trust in immunization. Pediatrics 2008;122:149-53.

8. Bosman RJ, Rood E, Oudemans-van Straaten HM, et al. Intensive care information system reduces documentation time of the nurses after cardiothoracic surgery. Intensive Care Med 2003;29:83-90.

Correspondence to: Dr. Jeffrey C. Kwong, Institute for Clinical Evaluative Sciences, Rm. G1-06, 2075 Bayview Ave., Toronto ON M4N 3M5; jeff.kwong@utoronto.ca

\section{Public Health Agency of Canada/Canadian Institutes of Health} Research Influenza Research Network Vaccine Coverage Theme Group: Writing team: Jeffrey C. Kwong MD MSc, Institute for Clinical Evaluative Sciences, Toronto, Ont.; Julie Foisy, MHSc, Ontario Agency for Health Protection and Promotion, Toronto, Ont.; Sherman Quan BSc, University Health Network, Toronto, Ont.; Christine Heidebrecht MSc, Ontario Agency for Health Protection and Promotion, Toronto, Ont.; Faron Kolbe MSc, Toronto Public Health, Toronto, Ont.; Julie A. Bettinger PhD MPH, Vaccine Evaluation Center, University of British Columbia, Vancouver, BC; David L. Buckeridge MD PhD, McGill University, Montréal, Que.; Larry W. Chambers PhD, Elisabeth Bruyère Research Institute, Ottawa, Ont.; Natasha S. Crowcroft MD MSc, Ontario Agency for Health Protection and Promotion, Toronto, Ont.; Irfan A. Dhalla MD MSc, Keenan Research Centre of the Li Ka Shing Knowledge Institute, St. Michael's Hospital, Toronto, Ont.; Christopher A. Sikora MD MPH, School of Public Health, University of Alberta, Edmonton, Alta.; and Donald J. Willison ScD, and Jennifer A. Pereira PhD, Ontario Agency for Health Protection and Promotion, Toronto, Ont. Other group members: Nicole Boulianne MSc, Institut national de santé publique du Québec, Québec City, Que.; Stephanie Brien BSc, McGill University, Montréal, Que.; Shelley Deeks MD MHSc, Ontario Agency for Health Protection and Promotion, Toronto, Ont.; Regina Elliott RN BScN, Durham Region Health Department, Whitby, Ont.; Michael Finkelstein MD MHSc, Toronto Public Health, Toronto, Ont.; Maryse Guay MD MSc, Université de Sherbrooke, Sherbrooke, Que.; Donna Kalailieff BScN MEd, Niagara Region Public Health, Thorold, Ont.; Jane Nassif MD, Niagara Region Public Health, Thorold, Ont.; Susan Quach MSc, Ontario Agency for Health Protection and Promotion, Toronto, Ont.

\section{HYDROMORPH Contin'qi2h}

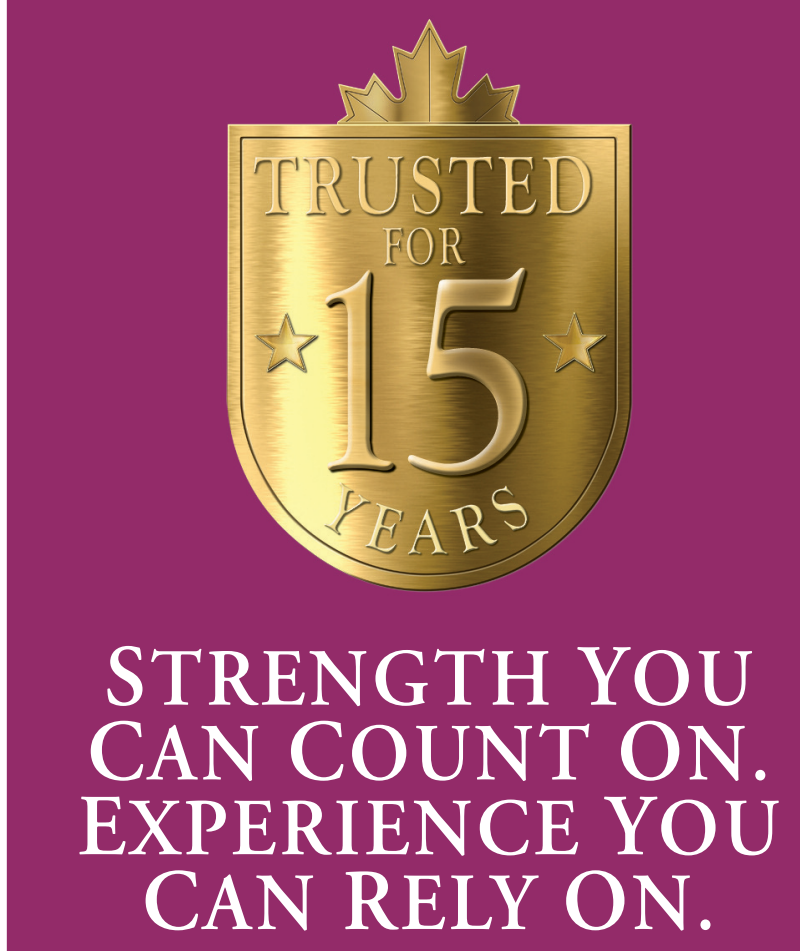

Hydromorph Contin ${ }^{\circledR}$ is indicated for the relief of severe chronic pain requiring the prolonged use of an oral opioid preparation.

Side effects are similar to other opioid analgesics. The most frequently observed are asthenic conditions, confusion, constipation, dizziness, lightheadedness, nausea, sedation, sweating and vomiting. Dosage limitations may be imposed by adverse effect. If they occur, please refer to prescribing information.

Warning: Opioid analgesics should be prescribed and handled with a high

degree of caution appropriate to the use of a drug with strong abuse potential. Patients should be cautioned not to consume alcohol while taking Hydromorph Contin ${ }^{\circledR}$, as it may increase the chance of experiencing dangerous side effects. Hydromorph Contin ${ }^{\circledR} 18 \mathrm{mg}$ capsules and higher are for use in opioid tolerant patients only. There is a potential for fatal respiratory depression in patients not previously exposed to similar equianalgesic doses of an opioid analgesic.

Hydromorph Contin ${ }^{\circledR}$ capsules or capsule beads should not be chewed, crushed or dissolved since this can lead to rapid release and absorption of a potentially fatal dose of hydromorphone. Product monograph available on request. Hydromorph Contin ${ }^{\circledR}$ capsule beads may be sprinkled on cold, soft food.

\section{'HYDROMORPH Contin'q'2h \\ Controlled release hydromorphone capsules}

A trusted first choice for severe chronic pain

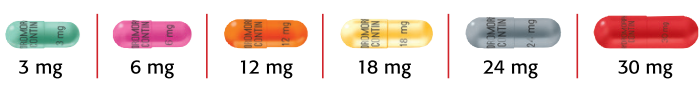

Initiation at $3 \mathrm{mg}$ q12h. Titration every 48 hours to satisfactory pain relief with acceptable side effects - increase first dose by $3 \mathrm{mg}$ to $6 \mathrm{mg}$ q $12 \mathrm{~h}$, then in $6 \mathrm{mg}$ increments from $12 \mathrm{mg}$ to 30 $\mathrm{mg} \mathrm{q} 12 \mathrm{~h}$ - with no ceiling dose.

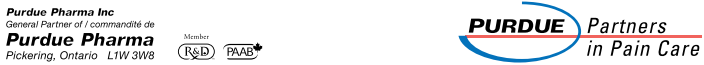

See prescribing summary on page 287 